\title{
Application of Grey System Theory in the Enterprise Supply Chain Collaboration Strategy
}

\author{
Wang Xin \\ Computer engineering college, Weifang University, Shandong 261061, China \\ www268@126.com
}

\begin{abstract}
In order to solve the problem of uncertain information in enterprises supply chain collaboration, a model of grey system theory is built to predict the coordination order quantity distributor of enterprise; the model has smaller prediction error and model accuracy. The data combined with empirical materials are calculated and simulated, and the simulation results are further improved analysis. The combination of qualitative and quantitative has a strong scientific application, which provides an important reference for distributors to determine the order and manufacturers to determine the optimal production in the supply chain, and the construction model and ideas of simulation analysis also have unique and innovative.
\end{abstract}

Keywords: Grey System Theory, Enterprise Supply Chain, Grey prediction, System Simulation

\section{Introduction}

Grey System Theory is a new cross-sectional discipline founded in 1982 by a famous Chinese scholar Professor Deng Julong. It uses the color depth to describe the clear degree of information, i.e. with the "black" means that information is unknown, with the "white" means the information entirely clear; with "grey" means part of the information clear, part of the information is not clear. Accordingly, the system information completely clear called "white system", the system information unknown called "black system", some information is clear, the partial information not explicit system known as the "grey system".

Modeling idea of grey system theory is a not very clear, the lack of information grey system from grey to white from the structure, model and relationship. With the uncertainty system of "partial information is known, some information is unknown," the "small sample", and the "poor information" for the study, and on the basis of information coverage, seeks practical rules through the sequence, which is characterized by "little data modeling", mainly through the "partial" information known to generate, develop, extract valuable information to achieve a correct understanding and effective control of the system behavior. Universal uncertainty about the poor information system of grey system theory, determines the new theory has a very broad prospects of development.

Just 20 years time, the rapid development of gray system theory not only in theory but also in the social sciences and natural sciences, which has been widely and in-depth applications in systems analysis, modeling, forecasting, and decision-making. The main contents include: the theory system based on Grey Hazy set, the analysis system based on grey relational space, the method system based on grey sequence generation, the model system based on grey modeling (GM) as the core, the technology system for the main of system analysis, evaluation, modeling, prediction, decision, control, and optimization.

Grey relational analysis method makes up the shortage of traditional statistical methods for system analysis result:

(1) Require large amounts of data, otherwise it is difficult to find out the statistics law. 
(2) Ask for a sample to a typical probability distribution, requirements between each factor data and system feature data showed a linear relation and the factors are independent of each other. This requirement is difficult to meet.

(3) Computing capacity, generally rely on computers to help.

(4) Quantitative results may be inconsistent with the qualitative analysis of the results, leading to the relationship and the law of system are distorted and upside down.

Thus, the method of gray system theory is an effective analysis design tool of supply chain collaboration strategy for high uncertainty businesses.

\section{Grey Prediction of Enterprises Order Quantity in Supply Chain}

In the grey forecasting model, an $n$ order, $h$ variable gray model called GM (n, h), As the predictive model generally consider only one variable, namely GM $(\mathrm{n}, 1)$, the $n$ value is greater, the greater amount of calculation, but the precision is not high, so $n$ generally is 1 , namely $\mathrm{GM}(1,1)$. The grey prediction model GM $(1,1)$ as an example to introduce how to use grey system theory to establish the mathematical model, and the model was improved, and finally test and evaluation of the precision of the predicted value of the model accuracy.

GM $(1,1)$ is a differential equation model of first order, a variable, suitable for the forecast value development of the size in system behavior changes. Its essence is through a cumulative generation of the original data sequence (1-AGO), the generated data sequence has certain regularity, thus structures the model.

(1) Using gray system theory to establish prediction model

GM $(1,1)$ means that there is only one variable, using the model of first order differential equations, the differential equation which represents:

$$
\frac{d X^{(1)}}{d t}+\alpha X^{(1)}=\beta
$$

Where: $\alpha, \beta$ - undetermined parameters, which $\alpha$ is called the development of gray number, $\beta$ as the endogenous control gray number.

$X^{(1)}$ - a cumulative generation of raw data $X^{(0)}, t$ - time.

The differential equation is an adaptable dynamic prediction model can reflect the continuity of the development.

(1) The collection of raw data

Original data are assumed:

$$
X^{(0)}=\left\{X^{(0)}(t) \mid t=1,2, \mathrm{~L} \quad \mathrm{~L} \quad n\right\}=\left\{X^{(0)}(1), X^{(0)}(2), \mathrm{L} \quad \mathrm{L}, X^{(0)}(n)\right\}
$$

(2) According to GM $(1,1)$ modeling method

First-order accumulated generation for known original data sequence $X^{(0)}$ (1-AGO):

$$
X^{(1)}(t)=\sum_{m=1}^{t} X^{(0)}(m)
$$

By generating sequences $X^{(1)}$ are as follows:

$$
\left.X^{(1)}=\left\{X^{(1)}(t) \mid t=1,2, \mathrm{~L} \quad \mathrm{~L} \quad n\right\}\right\}=\left\{X^{(1)}(1), X^{(1)}(2), \mathrm{L} \quad \mathrm{L}, X^{(1)}(n)\right\}
$$

(3) Construct data matrices $\mathrm{B}$ and data vector $Y_{N}$ 


$$
\begin{aligned}
& B=\left[\begin{array}{ll}
-\frac{1}{2}\left[X^{(1)}(1)+X^{(1)}(2)\right. & 1 \\
-\frac{1}{2}\left[X^{(1)}(2)+X^{(1)}(3)\right. & 1 \\
\mathrm{M} & \mathrm{M} \\
-\frac{1}{2}\left[X^{(1)}(n-1)+X^{(1)}(n)\right. & 1
\end{array}\right] \\
& Y_{N}=\left\{\begin{array}{l}
X^{(0)}(2) \\
X^{(0)}(3) \\
\mathrm{M} \\
X^{(0)}(n)
\end{array}\right]
\end{aligned}
$$

(4) Find the model parameters: $\alpha, \beta$

Let $\hat{a}=\left[\begin{array}{l}\alpha \\ \beta\end{array}\right]$ be the estimated parameter vector, the solution can be obtained using the least squares method:

$$
\hat{a}=\left[\begin{array}{l}
\alpha \\
\beta
\end{array}\right]=\left(B^{T} B\right)^{-1} B^{T} Y_{N}
$$

(5) Modeling

First-order grey differential equations $\operatorname{GM}(1,1)$ prediction model can be obtained as follows:

$$
\frac{d X^{(1)}}{d t}+\alpha X^{(1)}=\beta
$$

Solving the differential equation, get the time response equation of the model:

$$
\hat{X}^{(1)}(t+1)=\left(X^{(0)}(1)-\frac{\beta}{\alpha}\right) e^{-\alpha t}+\frac{\beta}{\alpha}
$$

Where: $t=0,1,2, \mathrm{~L} \mathrm{~L} \quad N$.

(2) Model improvement

To improve the accuracy and practical value of the model, the need for a second fitting parameter estimation, to further improve the model. The time response equation written as:

$$
\hat{X}^{(1)}(t+1)=A e^{-\alpha t}+B
$$

Where $t=0,1,2$, L L $N, A=\left(X^{(0)}(1)-\frac{\beta}{\alpha}\right)$, and $B=\frac{\beta}{\alpha}$.

Then according to the first estimate of $\alpha, \beta$ value and the original 1-AGO sequence $X^{(1)}(t)$ of A and B are estimated. Constructing data matrices $\mathrm{G}$ and data vector $X^{(1)}$ : 


$$
\begin{aligned}
& G=\left[\begin{array}{cc}
e^{0} & 1 \\
e^{-\alpha} & 1 \\
\mathrm{M} & \mathrm{M} \\
e^{-\alpha(n-1)} & 1
\end{array}\right] \\
& X^{(1)}=\left\{\begin{array}{ll}
X^{(1)}(1) \\
X^{(1)}(2) \\
M^{(1)}(n)
\end{array} \mid\right.
\end{aligned}
$$

Then find the parameters $\mathrm{A}$ and $\mathrm{B}$ :

$$
\left[\begin{array}{l}
A \\
B
\end{array}\right]=\left(G^{T} G\right)^{-1} G^{T} X^{(1)}
$$

To obtain the final time response equation:

$$
\hat{X}^{(1)}(t+1)=A e^{-\alpha t}+B
$$

This is a 1-AGO generation series forecasting model, predictive models can be drawn through the original series inverse accumulated generating IAGO, that prediction model is:

$$
\hat{X}^{(0)}(t+1)=\hat{X}^{(1)}(t+1)-\hat{X}^{(1)}(t)
$$

Where $t=0,1,2$, L L $N$ and $\hat{X}^{(0)}(1)=\hat{X}^{(1)}(1)$.

(3) Accuracy test of model and Accuracy assessment of prediction value

The most commonly used test model accuracy has residual test, correlation test and posterior error test, where the main use of the first and third test method for testing. The residual test is based on the original series and prediction model to calculate the simulated accumulated sequence, compute the residual sequence, and finally calculate relative error series. The arithmetic test method is simple and feasible, so it is widely used. Then the posterior error test is calculated variance of original series and residual series, and then according to their ratio and small error probability to judge accuracy.

First calculate the residuals:

$$
e(t)=X^{(0)}(t)-\hat{X}^{(0)}(t)
$$

where : $t=1,2, \mathrm{~L} \mathrm{~L} N$,

And then calculate the residual vector:

$$
e=(e(1), e(2), \mathrm{L} \mathrm{L} e(N))
$$

Indicate the variances of original series $X^{(0)}$ and residual Series e are $S_{1}{ }^{2}$ and $S_{2}^{2}$, then:

$$
S_{1}^{2}=\frac{1}{N} \sum_{t=1}^{N}\left(X^{(0)}(t)-\overline{X^{(0)}}\right)^{2}, \quad S_{2}^{2}=\frac{1}{N} \sum_{t=1}^{N}(e(t)-\bar{e})^{2},
$$

Calculate the posterior error ratio: 


$$
C=\frac{S_{2}}{S_{1}}
$$

A posteriori error ratio $\mathrm{C}$ is reflected in the accuracy of the model parameters, the smaller the better value $\mathrm{C}$. General Requirements for $\mathrm{C}<0.35$, the maximum is not more than 0.65 , and to evaluate the precision grade of model based the $\mathrm{C}$ value.

Then calculate the small error probability:

$$
P=p\left\{|e(t)-\bar{e}|<0.6745 S_{1}\right\}
$$

Small error probability $\mathrm{P}$ is a reflection of the accuracy of the model parameters, the $\mathrm{P}$ value is bigger is better. General requirements $\mathrm{P}>0.95$, the minimum cannot be less than 0.70 , and then evaluate the precision grade of model based the $\mathrm{P}$ value. The prediction precision grades by the posterior error test of the grey system theory are shown in table 1 .

Table 1. The Prediction Precision Grades Table by the Posterior Error Test

\begin{tabular}{ccccl}
\hline Grade & Good & Qualified & Reluctantly & Unqualified \\
\hline $\mathrm{C}$ & $<0.35$ & $<0.50$ & $<0.65$ & $\geq 0.65$ \\
$\mathrm{P}$ & $>0.95$ & $>0.80$ & $>0.70$ & $\leq 0.70$ \\
\hline
\end{tabular}

After the posterior error test, if the prediction precision is high, then the model is reliable, practical, do not need to set up a GM $(1,1)$ residual correction model, also has a higher forecasting accuracy. If the accuracy is not high, the model is not reliable, has a little value in practical application, need to set up $\operatorname{GM}(1,1)$ residual correction model.

\section{Examples of Simulation Modeling, Calculation and Analysis}

Whether for manufacturers or distributors of the supply chain, order quantity is very important data. If the manufacturer knows the order quantity of all its distributors, it could be reasonable to organize production, so as to reduce inventory, reduce production costs, and reduce the sales risk. If distributors know their optimal order quantity, then they can determine the quantity of each order and order cycle. Here the use of predictive models based on these established gray system theory to predict the enterprise's order quantity in supply chain. The reason for this approach is: As the impact of the economic order quantity development, consumer income levels, the price of the product itself, the price of related products, inventory levels and other factors, some of which are identified, and some factors are uncertain, so it can be regarded as a grey system. Meanwhile grey prediction method can effectively avoid the fatal weakness of lack of relevant data, but also to avoid the influence of personal experience, knowledge and preferences as well as macroeconomic policies and other factors caused by subjective and can better grasp the system's self evolution.

Example: Silver Spring Water Co. Ltd. is a small pure water production enterprise, the production of pure water sales in a chain supermarket. Here's to the supermarket in 2012 January to May order quantity to predict the order quantity from June to December.

(1) Establish the related model

(1) The collection of raw data

Here the original data used in the study were from cooperative distributors of Silver Spring limited liability Company -- a supermarket limited liability company collected data, which have analyzed and adjusted, see table 2 . 
Table 2. The Order Quantity in a Supermarket 2010 from January To May (Unit: Million Boxes)

\begin{tabular}{rccccc}
\hline Month & Januar & February & March & April & May \\
\hline $\begin{array}{c}\text { Order } \\
\text { quantity }\end{array}$ & 11.8 & 12.0 & 12.3 & 12.6 & 13.7 \\
\hline
\end{tabular}

Here in January as the starting point, i.e. the point $t=1$, then get the original data sequence:

$$
X^{(0)}=\left\{X^{(0)}(t) \mid t=1,2, \text { L L } 5\right\}=\left\{X^{(0)}(11.8,12.0,12.3,12.6,13.7)\right\}
$$

(2) According to GM $(1,1)$ modeling method

First-order accumulated generation for known original data sequence $X^{(0)}$ (1-AGO):

$$
X^{(1)}(t)=\sum_{m=1}^{t} X^{(0)}(m)
$$

By generating sequences $X^{(1)}$ are as follows:

$$
X^{(1)}=\{11.8,23.8,36.1,48.7,62.4\}
$$

(3) constructing data matrices B and data vector $Y_{N}$

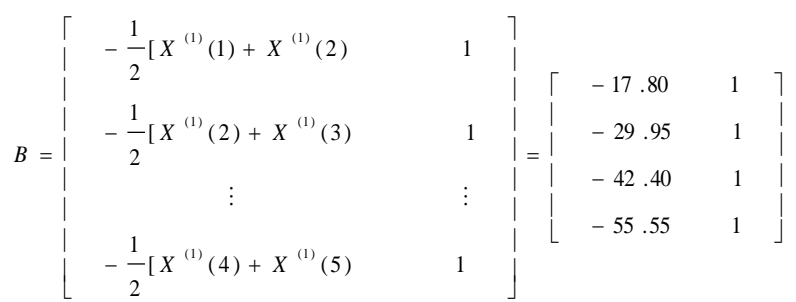

$$
Y_{N}=\left[\begin{array}{c}
X^{(0)}(2) \\
X^{(0)}(3) \\
\vdots \\
X^{(0)}(5)
\end{array}\right]=\left[\begin{array}{l}
12.0 \\
12.3 \\
12.6 \\
13.7
\end{array}\right]
$$

(4) Find the model parameters $\alpha, \beta$

Matrix calculation with EXCEL, we have:

$$
\hat{a}=\left[\begin{array}{l}
\alpha \\
\beta
\end{array}\right]=\left(B^{T} B\right)^{-1} B^{T} Y_{N}=\left[\begin{array}{c}
-0.0429805 \\
11.08198813
\end{array}\right]
$$

(5) Establish order quantity prediction model

First-order grey differential equations GM $(1,1)$ prediction model can be obtained as follows:

$$
\frac{d X^{(1)}}{d t}-0.0429805 X^{(1)}=11.08198813
$$

Solving the differential equation, get the time response of the model equation:

$$
\hat{X}^{(1)}(t+1)=269.637430175 e^{0.0429805 t}-257.837430175
$$


Where: $t=0,1,2$, L L $N$

(2) Improved model

To improve the accuracy and practical value of the model, the need for a second fitting parameter estimation, to further improve the model. The above time response equation is written:

$$
\hat{X}^{(1)}(t+1)=A e^{0.0429805 t}+B
$$

Where: $t=0,1,2$, L L $N$

Then according to the first estimate of the $\alpha, \beta$ value and the original 1-AGO series $X^{(1)}(t)$ on A and B estimation. Constructing data matrices $\mathrm{G}$ and data vector $X^{(1)}$ :

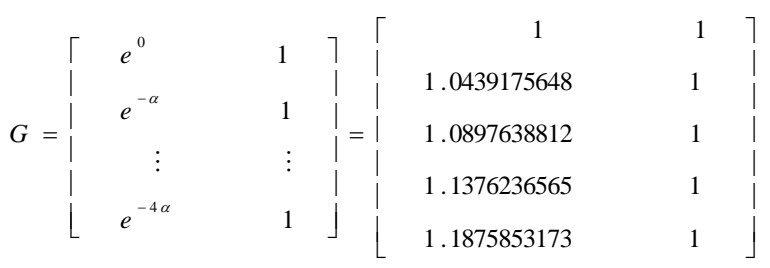

$$
X^{(1)}=\left[\begin{array}{l}
X^{(1)}(1) \\
X^{(1)}(2) \\
\vdots \\
X^{(1)}(5)
\end{array}\right]=\left[\begin{array}{l}
11.8 \\
23.8 \\
36.1 \\
48.7 \\
62.4
\end{array}\right]
$$

Then find the parameters $\mathrm{A}$ and $\mathrm{B}$ :

$$
\left\lceil\begin{array}{l}
A \\
B
\end{array}\right]=\left(G^{T} G\right)^{-1} G^{T} X^{(1)}=\left[\begin{array}{c}
268.89343416 \\
-257.01395825
\end{array}\right]
$$

To obtain the final time response equation:

$$
\hat{X}^{(1)}(t+1)=268.89343416 e^{0.0429805 t}-257.01395825
$$

This is a forecasting model of 1-AGO generation series, the predictive models can be drawn through the original series inverse accumulated generating IAGO, is an order quantity of forecasting model:

$$
\hat{X}^{(0)}(t+1)=\hat{X}^{(1)}(t+1)-\hat{X}^{(1)}(t)=268.89343416 \times e^{0.0429805} \times\left(1-e^{-1}\right)
$$

Where: $t=0,1,2, \mathrm{~L} \quad \mathrm{~L} \quad N, \hat{X}^{(0)}(1)=\hat{X}^{(1)}(1)$.

(3) Testing of the accuracy and precision assessment of the predicted value

Here mainly uses testing method of residual test and posterior error test.

First calculate the residuals:

$$
e(t)=X^{(0)}(t)-\hat{X}^{(0)}(t) \quad \times
$$

Where: $t=1,2, \mathrm{~L}$ L 5 . 
Residual vector is obtained:

$$
e=(-0.07947591,0.19085531 \quad,-0.02777356 \quad,-0.26917935,0.25563764)
$$

Indicate the variances of original series $X^{(0)}$ and residual Series e are $S_{1}^{2}$ and $S_{2}^{2}$, then:

$$
\begin{gathered}
S_{1}^{2}=\frac{1}{N} \sum_{t=1}^{N}\left(X^{(0)}(t)-\overline{X^{(0)}}\right)^{2}=0.440736258 \\
S_{2}^{2}=\frac{1}{N} \sum_{t=1}^{N}(e(t)-\bar{e})^{2}=0.036067974
\end{gathered}
$$

Calculate the posterior error ratio:

$$
C=\frac{S_{2}}{S_{1}}=0.28615<0.35
$$

So according to the ratio $\mathrm{C}$ of the posterior variance to assess the accuracy class of model is good.

Then calculate the small error probability:

$$
P=p\left\{|e(t)-\bar{e}|<0.6745 \quad S_{1}\right\}=100 \%>95 \%
$$

So according to the small error probability $\mathrm{P}$ to evaluate the precision grade of model is also good.

After the posterior variance testing, the model is reliable, practical, high prediction accuracy, do not need to set up a GM $(1,1)$ residual correction model, also has a higher

\begin{tabular}{|c|c|c|c|c|c|}
\hline Month & $\mathrm{y}^{\mathrm{J}}$ & February & March & April & May \\
\hline $\begin{array}{l}\text { The actual } \\
\text { order } \\
\text { quantity }\end{array}$ & 11.8 & 12.0 & 12.3 & 12.6 & 13.7 \\
\hline $\begin{array}{l}\text { Predicted } \\
\text { order } \\
\text { quantity } \\
\end{array}$ & $52^{11.879}$ & $6^{11.8091}$ & 12.32789 & 12.86928 & $4^{13.4344}$ \\
\hline $\begin{array}{l}\text { Absolute } \\
\text { error }\end{array}$ & $2^{0.0795}$ & -0.19084 & 0.02789 & 0.26928 & -0.26556 \\
\hline $\begin{array}{l}\text { Relative } \\
\text { error }\end{array}$ & $\%$ & $-1.602 \%$ & $0.0235 \%$ & $2.146 \%$ & $-1.872 \%$ \\
\hline
\end{tabular}
forecasting accuracy. Using the above model, the actual verification of historical data in Table 2, the prediction results and error analysis are shown in table 3.

Table 3. Accuracy Test of Order Quantity Model Prediction (Unit: Million Boxes)

\section{Conclusions}

Based on the application error analysis of order quantity prediction in collaboration with distributors of the model GM $(1,1)$, this paper established GM $(1,1)$ model is correct and reasonable. As the grey system theory is not starting from a statistical law, and therefore requires less raw data, the outstanding advantage of grey prediction model to make a viable, reliable method and means of enterprise collaboration vendor to predict 
order quantity. This model is another important goal lies in the enterprise through the ordering quantity prediction, select the suitable order model to organize the order, so that the total cost minimum, to achieve collaboration of the demand and supply in the supply chain, so as to achieve the best economic benefit.

\section{Acknowledgements}

This research is supported by the National Spark Program Project (2013GA740109), the Spark Program Project of Shandong Provence (2012XH06013) and the Weifang Science and Technology Development Plan Project (No. 2014GX021).

\section{References}

[1] L. Zhou, "The supplier in the supply chain-Channel Coordination Mechanisms between retailers", Industrial Engineering and Management, vol.4, (2010), pp. 36-38.

[2] S. H. Suo and Y. H. Jin, "Game analysis of the two stage supply chain between enterprises", Computer integrated manufacturing system-CIMS, vol. 9, no. 7, (2008), pp.16-19.

[3] L. W. Liu, "Partnership in the supply chain of enterprises", Computer integrated manufacturing systemCIMS, vol. 7, no. 8, (2009), pp. 21-22.

[4] D. Y. Mu, "Analysis of supply chain decisions based on game theory", Nankai University, (2009), pp. 56-62.

[5] Q. Jin and T. Su, "Study on coal demand forecasting model based on grey theory", Journal of Shandong Science and Technology University, vol. 3, (2004), pp. 44-46.

[6] B. Wang and T. J. Yang, "Supply chain model of production and pricing game theory under stochastic demand", Industrial Engineering, vol. 11, (2006), pp. 13-15.

[7] Q. L. Gu and T. G. Gao, "Pricing Strategy Analysis for Reverse Supply Chain Based on Game Theory", Systems Engineering Theory and Practice, vol. 3, (2005), pp. 25-27.

[8] F. L. Zhang and F. Shi, "The stochastic grey system model to forecast the passenger and freight volume", Journal of Central South University, vol. 2, (2009), pp. 32-33.

[9] T. M. John, D. William, S. K. James, M. Soonhong, W. N. Nancy, D. S. Carlo and G. Z. Zach, "Defining supply chain management", Journal of Business Logistics, vol. 22, no. 2, (2002), pp. 1-26.

[10] E. Sandbcrg, "Logistics collaboration in supply chains-a survey of Swedish manufacturing companies", Department of Management and Economics, Linkoping University, Linkoping, vol. 5, (2005), pp. 180121.

[11] F. Finley and S. Srikanth, "Imperatives for Successful Collaborative", Supply chain Management Review, vol. 9, no. 1, (2005), pp. 30-37.

[12] A. Vim, "Logistics Flows coordination in supply chains Using Enterprise Input-output Models", Proceeding of the IEEE International Conference on Industrial Engineering and Engineering Management, (2007) , December 2-4, pp. 1603-1608, Singapore.

[13] H. Selim, C. Araz and I. Ozkarahan, "Collaborative production-distribution planning in supply chain: A fuzzy goal programming approach", Transportation Research Part E, vol. 44, (2008), pp. 396-419.

[14] G. N. Nyaga, J. M. Whipple and D. F. Lynch, "Examining supply chain relationships: Do buyer and supplier perspectives on collaborative relationships differ?", Journal of Operation Management, vol. 28, No. 2, (2010), pp. 101-114.

\section{Author}

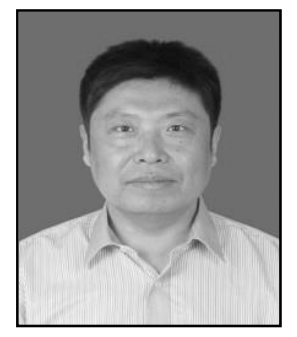

Wang Xin, He received his master's degree in Computer Application Technology from Shandong University of Science and Technology in 2004. Currently he is a vice professor at the school of computer engineering in Weifang University, China. He is a member of China Computer Federation. His current research interests include computer network, computational intelligence and computer communication. He has published 4 books, and more than 50 research papers in journals and international conferences. 
International Journal of Grid Distribution Computing Vol. 8, No.4, (2015) 